\title{
Discriminação de fitofisionomias de floresta de várzea a partir do algoritmo Iterated Conditional Modes aplicado aos dados SAR/R99 (QUAD-POL/Banda L)
}

\author{
Gustavo Manzon NUNES'르, Carlos Roberto de SOUZA FILHO², Laerte Guimarães FERREIRA³ \\ RESUMO \\ Utilizando-se dados do sensor aerotransportado SAR R99, adquiridos na banda L (1,28 GHz) em amplitude e com quatro \\ polarizaçóes $(\mathrm{HH}, \mathrm{VV}, \mathrm{HV}$ e VH), avaliou-se a distinção de fitofisionomias de floresta de várzea existentes nas Reservas de \\ Desenvolvimento Sustentável Amanã e Mamirauá e áreas adjacentes, com a aplicação do algoritmo Iterated Conditional Modes \\ (ICM) de classificação polarimétrica pontual/contextual. Os resultados mostraram que o uso das distribuiçóes multivariadas \\ em amplitude, conjuntamente com uma banda de textura, produziu classificaçóes de qualidade superior àquelas obtidas com \\ dados polarimétricos uni/bivariados. Esta abordagem permitiu a obtenção de um índice Kappa de 0,8963, discriminando \\ as três classes vegetacionais de interesse, comprovando assim o potencial dos dados do SAR R99 e do algoritmo ICM no \\ mapeamento de florestas de várzea da Amazônia.
}

PALAVRAS-CHAVE: Radar de Abertura Sintética, Sensoriamento Remoto, Mapeamento de Florestas Tropicais.

\section{Discrimination among flooded forest phytophysiognomies from Iterated Conditional Modes algorithm applied to SAR Data R99 (QUAD POL/L-Band)}

\begin{abstract}
This study seeks to evaluate the capability of data generated by the synthetic aperture radar SAR R99 sensor to map phytophysiognomies found in the Amaná and Mamirauá Sustainable Development Reserves (RDSA and RDSM). By means of L-band (1.28 GHz), full polarimetric ( $\mathrm{HH}, \mathrm{VV}, \mathrm{VH}, \mathrm{HV})$, amplitude data acquired with the SAR R99 sensor, distinctions among flooded forest phytophysiognomies in the RDSA and RDSM and around were achieved. The Iterated Conditional Modes (ICM) algorithm was employed to perform the local/contextual polarimetric classification of the data. Results showed that the use of multivariate distributions in amplitude with a band of texture produced classifications of superior quality in relation to those obtained with the uni/bivariate polarimetric data. This approach allowed to obtain a Kappa index of 0,8963 and the distinction of three vegetation classes of interest, demonstrating the potential of SAR R99 and the ICM algorithm to map flooded vegetation of the Amazon.
\end{abstract}

KEYWORDS: synthetic aperture radar, remote sensing, tropical forest mapping.

1 UNIVERSIDADE FEDERAL DE MATO GROSSO. gustavomn@gmail.com

2 UNIVERSIDADE ESTADUAL DE CAMPINAS. beto@ige.unicamp.br

${ }^{3}$ UNIVERSIDADE FEDERAL DE GOIÁS. laerte@iesa.ufg.br 


\section{INTRODUÇÃO}

Nas últimas duas décadas, houve um grande desenvolvimento da tecnologia SAR com consequências importantes para estudos da vegetação localizada, principalmente, em ambientes tropicais e boreais. As variaçóes na estrutura da vegetação, a existência do conteúdo hídrico na massa foliar, a presença de solo exposto e suas condicionantes físicas e estruturantes, influenciam o retorno do sinal emitido por radares de abertura sintética (SAR), por exemplo, nas bandas $\mathrm{C}$ e $\mathrm{L}$ (comprimentos de onda em torno de $5 \mathrm{~cm}$ e $23 \mathrm{~cm}$, respectivamente) (Kasischke et al. 1997; de Jong et al. 2000; Narvaes et al. 2010). Radares que operam em banda L (frequência de 1,28 GHz) têm ainda a capacidade de penetração parcial no dossel e, consequentemente, interação com troncos e galhos (Saatchi et al. 2001; Rosenqvist et al. 2003; Santos et al. 2009; Narvaes et al. 2010).

Para a banda L, as polarizaçōes $\mathrm{HH}$ e VV fornecem a melhor penetração através do dossel da vegetação, enquanto as polarizaçôes cruzadas $\mathrm{HV} \mathrm{e} \mathrm{VH} \mathrm{fornecem} \mathrm{mais} \mathrm{informaçôes}$ sobre a estrutura do dossel florestal. (Rosenqvist et al. 2007). Da mesma forma, radares que operam em banda $\mathrm{C}$ também têm sido utilizados para o delineamento de comunidades com vegetação baixa; como os sinais de ondas curtas interagem principalmente com as folhas e galhos no topo da vegetaçáo dossel (Lucas et al. 2004), esta banda pode ser utilizada em ambiente de zona úmida para delinear vegetação herbácea nativa (e.g. Costa et al. 2002) e cultivada (e.g., Le Toan et al. 1997).

Os sistemas SAR que operam em banda L (JERS-1, ALOS-PALSAR) são sensíveis às propriedades estruturais da vegetação. Como as folhas das árvores geralmente são muito pequenas para serem detectadas, os sinais em banda $\mathrm{L}$ interagem com os ramos, bem como fornecem informaçóes sobre o tronco e o contato com a superfície do terreno. Esta propriedade faz com que o uso de dados SAR em banda L forneça informaçôes particularmente significativas no contexto das zonas úmidas, possibilitando a distinção entre floresta inundada e floresta de terra firme (Krohn et al. 1983; Hess et al. 2003).

A utilização de dados SAR em banda L para o mapeamento e monitoramento de zonas úmidas em escalas regionais teve início com o satélite JERS-1, através do Projeto Global Forest Mapping (GRFM / GBFM). Esse projeto serviu para estimular a aquisição de séries temporais consistentes, com mais de duas coberturas anuais, visando caracterizar a dinâmica espaçotemporal e a complexidade dos ambientes de floresta em áreas boreais e tropicais. (Rosenqvist et al. 2000, 2004a).

A partir da experiência adquirida durante o projeto GRFM / GBFM, outra iniciativa internacional, denominada Kyoto \& Carbon (K\&C), foi iniciada em 2006, com o intuito de apoiar estudos vinculados ao ciclo do carbono e às questóes ambientais. Esse projeto, liderado pela JAXA, baseia-se nos dados gerados pelo ALOS PALSAR em banda L (Rosenqvist et al. 2007). Assim, em consonância com o tópico envolvendo as Zonas Úmidas do K\&C, o sensor PALSAR vem fornecendo dados consistentes e com repetidas observaçôes em banda L, compreendendo duas polarizações e resolução espacial de 10 a 20 metros, possibilitando a caracterização e o acompanhamento das perturbaçóes nos ecossistemas (Rosenqvist et al. 2004b).

As potencialidades dos dados SAR R99 em aplicaçôes na área florestal e ambiental podem ser verificadas em recentes estudos, como os realizados por Gonçalves (2007), na estimativa volumétrica para áreas de floresta tropical; Miranda et al. (2007), na classificação textural de tipologias da floresta alagada; Fernandez (2007), no mapeamento de corte seletivo; Gualberto e Souza Filho (2007), no reconhecimento de ambientes costeiros a partir de análise textural e pelo SIPAM (2007), no monitoramento da ação antrópica em áreas especiais.

O objetivo desse estudo é demonstrar a potencialidade dos dados do sensor aerotransportado SAR R99 em banda L associada à utilização do algoritmo de classificação ICM, visando à distinção de fitofisionomias de floresta de várzea existentes em ambientes da Reserva de Desenvolvimento Sustentável Mamirauá (RDSM), Amanã (RDSA) e áreas adjacentes.

\section{MATERIAL E MÉTODOS}

\section{Área de estudo e base de dados}

Foram utilizadas imagens do sensor aerotransportado SAR R99, obtidas em banda L nas polarizaçóes $\mathrm{HH}, \mathrm{HV}$ e VV, com resolução espacial de seis metros. O sensor SAR é operado pelo Segundo do Sexto Grupo de Aviação (2\%/6 $\mathrm{GAv}$ ) da Força Aérea Brasileira (FAB), tendo como principais características o suporte a missōes de vigilância, mapeamento ou missōes híbridas de vigilância/mapeamento na Amazônia Legal. Estas imagens, em amplitude, foram obtidas no dia 02 de junho de 2005. A programação e o modo de imageamento do sensor SAR foram delimitados em função da característica da área e do objetivo do estudo, abrangendo ecossistemas de floresta alagada da RDSM, da RDSA e áreas adjacentes (Tabela 1). Porém, para o trabalho aqui realizado, devido à exigência computacional dos processamentos digitais dos dados SAR, foi selecionada uma área-piloto existente em um dos segmentos (segmento 3) que pode ser observada na Figura 1, compreendendo fitofisionomias de floresta de várzea (restinga baixa, restinga alta e chavascal), além da classe de lâmina d'água.

Para apoiar o trabalho, também foram utilizadas as cartas de cobertura vegetal do Bioma Amazônico do PROBIO, 
Tabela 1 - Características do imageamento SAR R99 sobre a área de estudo.

\begin{tabular}{lc}
\hline Parâmetros & Especificações \\
\hline Data da coleta & $02 / 06 / 2005$ \\
\hline Modo de Imageamento & Quad L + X \\
\hline Polarizações & $\mathrm{HH}, \mathrm{HV}, \mathrm{VV}, \mathrm{VH}$ \\
\hline Tipo de dado & Unsigned 8 bits \\
\hline Número de Looks & 8 Looks \\
Resolução da imagem & 6 metros \\
\hline Tamanho do pixel & 5 metros \\
Número de segmentos & 10 segmentos \\
Sistema de Coordenadas & Geográfica (lat/long) \\
Datum (Sistema Geodésico) & WGS-84 \\
Largura da Faixa Imageada & 20 km \\
Ângulo de Incidência & $36^{\circ}-83^{\circ}$ \\
Altitude de vôo & $11.000 \mathrm{~m}$ \\
Sentido de vôo & $\mathrm{N}-\mathrm{S}, \mathrm{S}-\mathrm{N}$ \\
\hline
\end{tabular}

na escala 1:250.000 (2006). A classificação digital através do algoritmo ICM foi gerada a partir do fragmento do segmento 3 da missão realizada. As imagens SAR R99 foram georreferenciadas, convertidas para a projeção "ground range", quantificadas em 16 bits, realçadas via contraste linear e convertidas para 8 bits. Esse trabalho de pré-processamento foi realizado nos laboratórios do SIPAM no Centro Regional de Manaus, utilizando rotinas padrão.

\section{Tipos fisionômicos da vegetação de várzea}

A várzea da RDSM e da RDSA, bem como a maior parte da vegetação das várzeas do baixo Rio Japurá, é composta, principalmente, por três fisionomias de grande importância segundo o perfil longitudinal apresentado na Figura 2. Duas destas fisionomias encontram-se em áreas de restinga e a terceira é denominada chavascal.

A restinga baixa corresponde à transição das áreas florestais da várzea para o chavascal e representa quase $85 \%$ da cobertura florestal da várzea amazônica, estabelecendo-se onde a média anual da coluna d'água é maior que 3 metros, com período de inundaçáo anual maior que 50 dias por ano, sendo seu sub-bosque frequentemente mais limpo e com boa visibilidade. (Wittmann et al. 2002; Ayres 2006). Já a restinga alta corresponde aos terrenos mais elevados da várzea. Estas terras estão sujeitas à inundação anual por dois a quatro meses, com profundidade que varia entre 1 a 2,5 metros. As restingas altas representam cerca de $12 \%$ da área florestal da várzea amazônica. Embora sejam estruturalmente mais parecidas com a vegetação de terra firme, a composição de espécies é muito diferente (Ayres 2006).

A fisionomia do chavascal consiste em áreas bastante extensas de vegetação baixa, arbustiva, pantanosa e quase impossível de ser transposta durante a seca. O chavascal é inundado anualmente durante cerca de seis a oito meses, a uma profundidade de 6 a 7 metros. No meio desta vegetaçáo arbustiva, pode-se observar a presença de algumas árvores emergentes ou até mesmo pequenas ilhas de restinga baixa. (Ayres 2006).

\section{Metodologia Utilizada}

A etapa metodológica de análise dos dados foi dividida em: (a) pré-processamento das imagens, (b) processamento digital, (c) reavaliação/integraçáo. A fase de pré-processamento

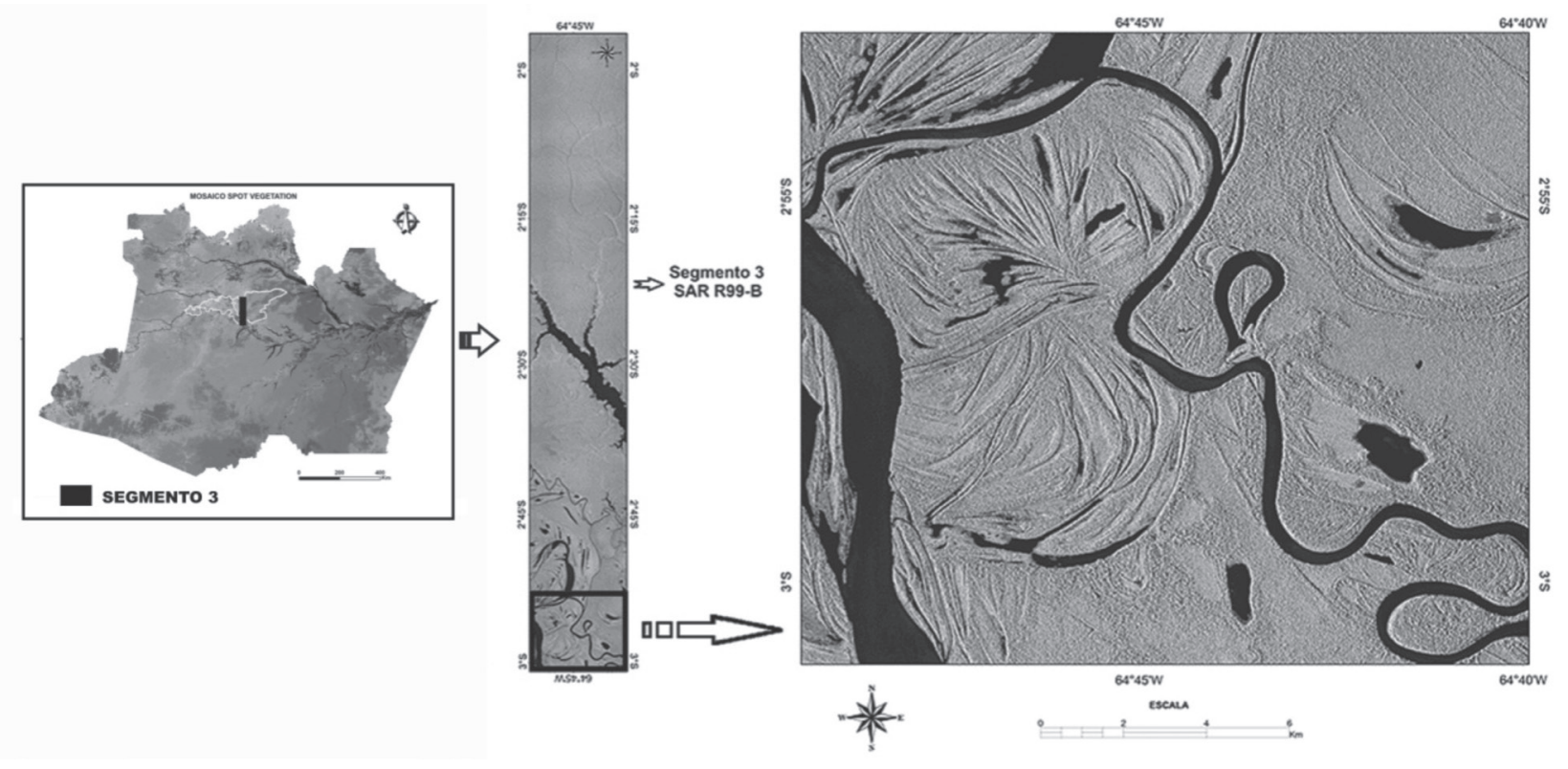

Figura 1 - Localização da área selecionada no segmento 3 onde foram realizados os processamentos digitais. 


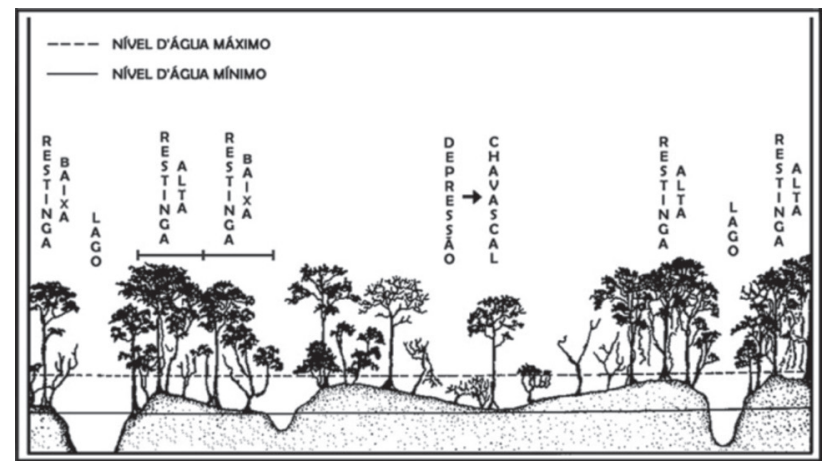

Figura 2 - Esquema de uma secção longitudinal representando a várzea da área em estudo. Fonte: Adaptado de Ayres (2006).

envolveu correções geométricas e radiométricas, incluindo a correçáo do padrão da antena. Na Figura 3, são apresentadas cada uma das etapas desenvolvidas.

A calibração radiométrica do segmento onde se situava a área em estudo consistiu na correção do padrão da antena de acordo com o ganho do sensor utilizado, reduzindo assim a influência da variaçáo do ângulo de incidência no retroespalhamento dos alvos. Utilizou-se o modelo multiplicativo, por meio de uma funçáo de ajuste obtida a partir de áreas homogêneas da imagem que se estendiam do alcance próximo ao alcance distante (Ulaby et al. 1982).

A calibração geométrica envolveu o registro do segmento contendo a área em estudo, tendo-se como base os mosaicos do sensor ETM+ do Landsat-7 ortorretificados da NASA (GeoCover).

O processamento digital envolveu a geração de composiçóes coloridas das bandas polarizadas, além da aplicação do algoritmo ICM desenvolvido por Vieira (1996) e implementado por Correia (1999) na linguagem IDL, o que permite sua aplicação no ambiente do software ENVI.

A utilizaçáo do algoritmo ICM para o processamento de dados SAR R99 tem obtido bons resultados que podem ser consultados em trabalhos já realizados, podendo-se citar mais recentemente estudos visando o mapeamento do uso e cobertura da terra em áreas da floresta amazônica (Freitas et al. 2008), como também análises objetivando a discriminação de incrementos de desflorestamento na Amazônia (Guerra et al. 2010).

O algoritmo ICM pressupóe uma forma integrada da classificação pontual com a contextual (respectivamente, classificação MaxVer e ICM), compreendendo um método iterativo de refinamento de classificaçóes que consiste em substituir a classe associada a cada coordenada pela classe que maximiza um determinado critério (Vieira 1996).

O classificador de máxima verossimilhança é um método de classificação pixel a pixel no qual dado uma imagem com
$\mathrm{N}$ pixels, cada pixel (descrito pelo seu vetor de atributos $\mathrm{x}$ ) será classificado como sendo da classe $\omega_{1}$ se

$g_{j}(x)>g_{k}(x)$ para todo $j \neq k, j, k=1, \ldots, N$

em que $\mathrm{g}_{\mathrm{j}}$ é denominada função discriminante para a classe $\omega_{i}$.

Para o classificador MAXVER, onde os dados seguem uma distribuição gaussiana multivariada, a função discriminante pode ser definida como:

$$
\mathrm{g}_{\mathrm{j}}(\mathrm{X})=-\frac{1}{2}\left(x-m_{j}\right)^{t} \sum_{j}^{-1}\left(x-m_{j}\right)-\frac{1}{2} \ln \left|\sum_{j}\right|+\ln P\left(\omega_{j}\right)
$$

em que $m_{j}$ e $\sum_{i}$ correspondem ao vetor de média e a matriz de covariância e $\mathrm{P}(\omega)$ é a probabilidade a priori da classe $\mathrm{j}$. As médias e matrizes de covariância das classes de interesse são estimadas a partir de amostras conhecidas. As probabilidades

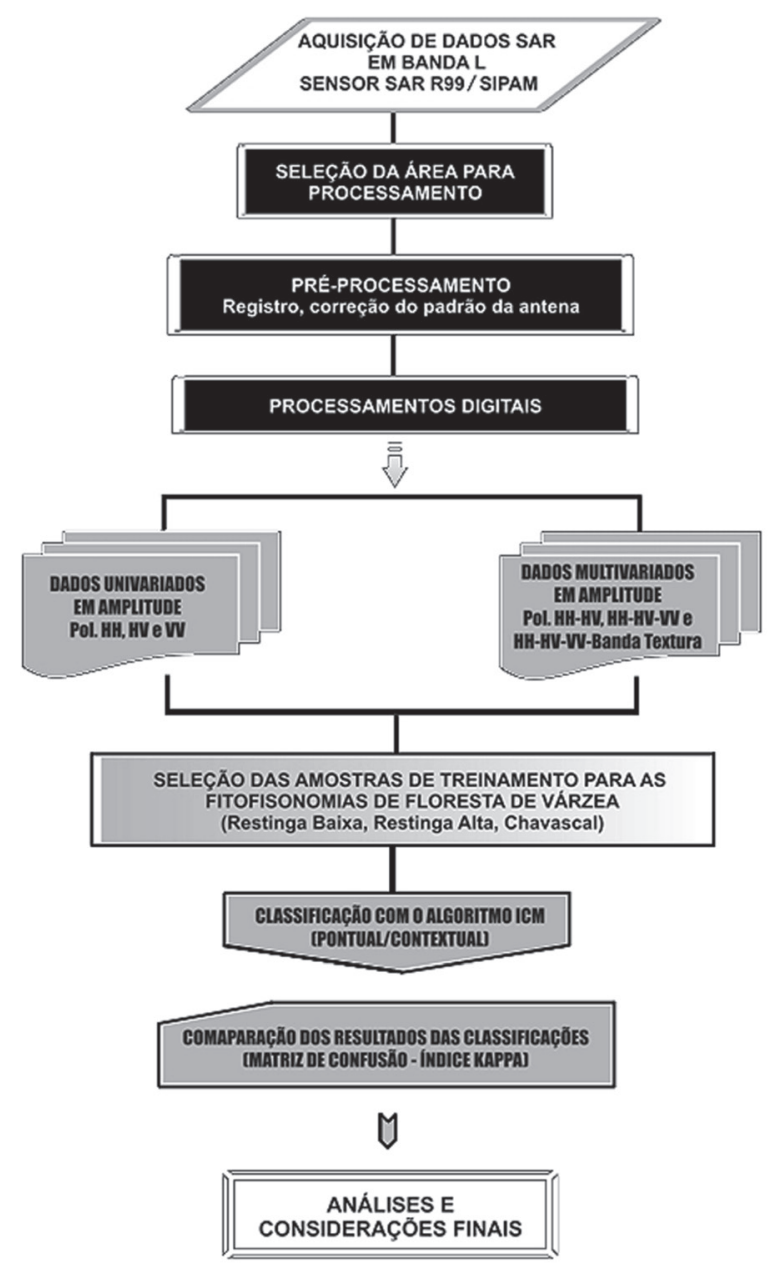

Figura 3 - Etapas metodológicas desenvolvidas, referentes às análises nos dados do sensor SAR R99. 
a priori são normalmente consideradas iguais e desprezadas no cálculo da funçâo de comparação (Dutra e Huber 1999). No entanto, para melhorar o resultado da classificação é necessário um classificador que, além de considerar o vetor de observaçôes do pixel, incorpore a informação da vizinhança deste pixel. Um desses classificadores é o ICM (Correia 1999), o qual se baseia na teoria de campos markovianos (Aboud Neta et al. 2010). Deste modo, considerando iguais as probabilidades a priori de cada classe, a funçấo discriminante pode ser definida por:

$$
\mathrm{g}_{\mathrm{j}}(\mathrm{X})=-\frac{1}{2}\left(x-m_{j}\right)^{t} \sum_{j}^{-1}\left(x-m_{j}\right)-\frac{1}{2} \ln \left|\sum_{j}\right|+\beta \succ\left\{t \in \delta_{x}: \omega_{i}=\omega_{x}\right\}
$$

em que $\beta$ é um parâmetro real que quantifica a influência das classes vizinhas, sendo estimado iterativamente, $\succ$ representa o número de elementos de um conjunto, t denota a coordenada de um pixel e $\delta_{\mathrm{x}}$ é o conjunto de coordenadas em torno da posição de $\mathrm{x}$.

Visando aumentar o desempenho dos classificadores digitais utilizados, optou-se por obter uma banda de textura que se caracteriza por representar a distribuição dos níveis de cinza da imagem. A textura é uma importante característica utilizada na interpretaçâo visual de imagens, principalmente, em imagens SAR com alta resolução espacial.

Para a obtenção da banda de textura, foi utilizado um filtro de co-ocorrência, com dimensôes de 5 pixels x 5 pixels.

Utilizando-se o algoritmo ICM, foram realizadas as classificaçôes pontuais/contextuais, o que possibilitou a modelagem estatística dos dados SAR univariados (polarização $\mathrm{HH}, \mathrm{HV}$ e VV), em que cada classe é ajustada pela distribuição estatística que melhor lhe represente. O programa permite o ajuste às seguintes distribuiçóes: normal, raiz quadrada da Gama, amplitude K, amplitude G0, lognormal e Weibull (Vieira 1996).

A seleçáo das amostras de cada classe (10 para cada classe), que compreendem as fitofisionomias existentes na imagem, foi baseada em áreas visitadas durante a campanha de campo, tendo sido acompanhada por identificador botânico responsável pela identificação das espécies características de cada fitofisionomia estudada. A campanha de campo foi realizada no mês de junho de 2005, sendo este o mês do ano caracterizado pelo decréscimo do nível de pluviosidade na região. Foram coletadas amostras (polígonos) de forma que as condiçốes de aleatoriedade e de não correlação espacial entre os pixels fossem garantidas. $\mathrm{O}$ tamanho do conjunto das amostras para cada fitofisionomia variou entre 1.500 a 2.200 pixels, o que assegurou boa representatividade estatística.

Para os dados multivariados em amplitude, as classificaçôes utilizando o algoritmo ICM foram implementadas somente considerando a distribuiçấo normal multivariada para as classes. Assim, para verificar qual método apresenta o melhor resultado final, realizou-se a classificação das seguintes imagens bi/multivariadas:

Pol. HH-HV;

Pol. HH-HV-VV;

Pol. HH-HV-VV-banda de textura.

Para o classificador de Máxima Verossimilhança, utilizouse um limiar de aceitação de $100 \%$, assumindo-se assim que todos os pixels das amostras de treinamento fossem atribuídos a uma determinada classe. Os critérios utilizados nas classificaçôes ICM foram: mínimo de $0,1 \%$ de trocas de classe do pixel e máximo de 20 interaçóes. Isso significa que o classificador ICM realizou mudanças de classes dos pixels até que o percentual de mudança fosse menor que 0,1\% entre uma interação e outra ou até que o número de iterações alcançasse 20 .

Foram comparados tanto os resultados das classificações quanto o melhor método de seleção de atributos (univariados, bi/multivariados e mutivariados/textutrais), utilizando-se o classificador pontual MaxVer e o classificador contextual ICM.

Para as comparaçôes dos resultados, foram utilizados a matriz de confusão, o valor do Kappa e a variância do Kappa, em que foram considerados os intervalos especificados por Landis e Koch (1977) (Tabela 2). A matriz de confusāo foi construída através da informação de verdade das amostras de teste, isto é, supóe-se verdade que todos os pixels das amostras de teste pertencem às classes a que foram atribuídos. A avaliação da classificação, utilizando a matriz de confusão, foi feita pela proporção de amostras corretamente classificadas, denominada exatidão global, que corresponde à razáo entre a soma da diagonal da matriz de confusão (amostras corretamente classificadas) e a soma de todos os elementos desta matriz (número total de amostras). Deve-se salientar que a exatidão global e o índice Kappa fornecem a mesma informação para a ordenaçáo dos resultados (dos dois classificadores, amostras e variáveis consideradas), e apenas variam nos valores atribuídos à precisão absoluta da classificação.

Tabela 2 - Conceitos de eficiência do coeficiente Kappa.

\begin{tabular}{cc}
\hline Kappa & Conceito \\
\hline$<0$ & Péssima \\
$0<\mathrm{k} \leq 0,2$ & Má \\
$0,2<\mathrm{k} \leq 0,4$ & Razoável \\
$0,4<\mathrm{k} \leq 0,6$ & Boa \\
$0,6<\mathrm{k} \leq 0,8$ & Muito Boa \\
$0,8<\mathrm{k} \leq 1,0$ & Excelente \\
\hline
\end{tabular}

Fonte: Landis e Koch (1977)

\section{RESULTADOS E DISCUSSÃO}

Para um melhor entendimento, são apresentados primeiramente os resultados das classificaçóes dos dados 
univariados com as polarizaçóes $\mathrm{HH}, \mathrm{HV}$ e VH. Em seguida, são apresentados os resultados dos dados bivariados com as polarizaçóes $\mathrm{HH}-\mathrm{VV}$ e multivariados com as polarizaçóes HH-HV-VV e HH-HV-VV-banda de textura (TEX.).

\section{Dados univariados em amplitude}

Objetivando-se avaliar os resultados obtidos com os classificadores MaxVer e ICM, para os dados polarimétricos, utilizou-se como uma das bases de comparação os resultados das classificaçóes para os dados univariados (monoespectrais) em amplitude, através do uso das distribuiçôes mais ajustadas.

Para determinar os histogramas com a distribuição mais ajustada de cada classe, é importante salientar que foram consideradas como melhores distribuiçôes aquelas com os menores valores de probabilidade $(\mathrm{p})$ no teste $\mathrm{X}^{2}$ segundo o sistema do algoritmo ICM, melhor detalhado em Correia (1999). Os valores p mais baixos asseguram, para cada classe, que a correlação é significativa ao nível de confiança próximo a $100 \%(p-0)$. Salienta-se que o classificador polarimétrico ICM possibilita a modelagem estatística dos dados de SAR para distribuições apropriadas às imagens de radar, inclusive para a própria distribuiçáo Normal.

A partir das classificaçôes MaxVer e ICM aplicadas nas polarizaçōes em amplitude na banda L (Figura 4), foram obtidas as respectivas matrizes de confusão. Na Tabela 3 são apresentados os valores estimados do coeficiente de concordância Kappa $(\hat{\mathrm{k}})$, as respectivas variâncias $\left(\hat{\sigma}_{\mathrm{k}}^{2}\right)$ e os conceitos obtidos, segundo Landis e Koch (1977), para as classificaçóes MaxVer e ICM dos dados univariados em amplitude na banda L.

Considerando-se os dados univariados em amplitude das polarizaçóes na banda L, verificou-se, de modo geral, que:

Comparativamente às matrizes de confusão das classificações ICM, as matrizes de confusão das classificaçōes MaxVer apresentaram resultados inferiores tanto no índice de concordância Kappa quanto em relaçáo aos valores de variância e exatidão global;

As classificaçóes MaxVer HH, ICM HH, ICM HV e ICM VV atingiram o conceito "Muito Boa" segundo os intervalos de índice Kappa estabelecidos por Landis e Koch (1977);

Tabela 3 - Exatidão das classificações dos dados univariados em amplitude na banda $L$ segundo coeficiente de concordância Kappa $(\hat{\mathrm{k}})$ e variâncias $\left(\hat{\sigma}_{\mathrm{k}}^{2}\right)$.

\begin{tabular}{lccc}
\hline Classificação & $\hat{\mathbf{k}}$ & $\hat{\sigma}_{\mathbf{k}}^{\mathbf{2}}\left(\times 10^{-5}\right)$ & Conceito \\
\hline MaxVer HH & 0,6328 & 4,5527 & Muito Boa \\
MaxVer HV & 0,5466 & 5,2234 & Boa \\
MaxVer VV & 0,4872 & 5,5610 & Boa \\
ICM HH & 0,7358 & 3,5594 & Muito Boa \\
ICM HV & 0,6787 & 4,1754 & Muito Boa \\
ICM VV & 0,6983 & 4,0798 & Muito Boa \\
\hline
\end{tabular}

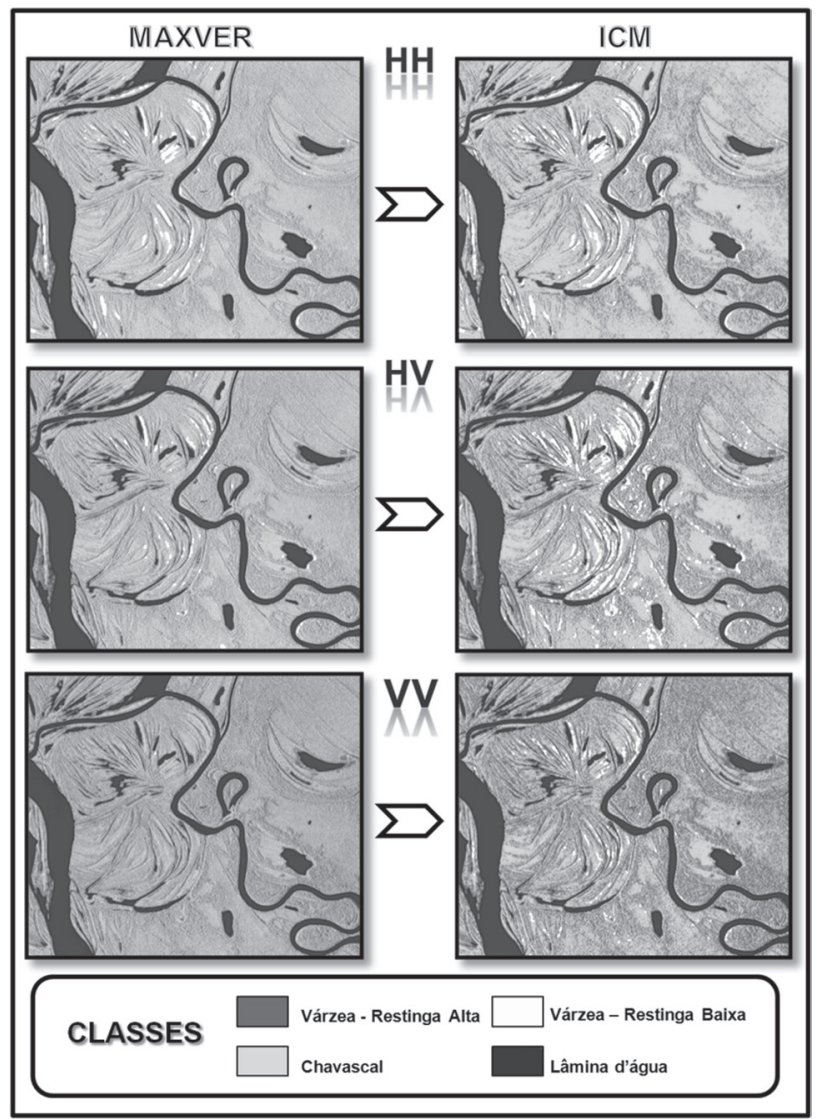

Figura 4 - Classificações geradas com os dados univariados em amplitude.

A melhor classificação foi obtida pelo método contextual ICM HH, obtendo-se um índice Kappa de 0,7358 e exatidáo global da classificação de 80,08 \%;

Comparando-se os dados da matriz de confusão da melhor classificação com os dados univariados (ICM HH) (Tabela 4), verificou-se que ocorreu maior confusão do classificador entre as classes fisionômicas do Chavascal e da Várzea - Restinga Alta, possivelmente, devido à polarização $\mathrm{HH}$ não fornecer a informação volumétrica e estrutural existente na vegetação de Restinga Alta.

\section{Dados multivariados em amplitude}

$\mathrm{Na}$ avaliaçáo dos resultados obtidos com os classificadores MaxVer e ICM para os dados polarimétricos multivariados, foram avaliadas classificaçóes com duas polarizaçóes, três polarizaçóes e com três polarizaçóes adicionadas a uma banda textural (contraste). Para este tipo de classificação, o programa foi implementado considerando-se uma distribuição normal multivariada para as classes; neste caso, náo é possível selecionar outro tipo de distribuição para a modelagem dos dados SAR. 
Tabela 4 - Matriz de confusão para a classificação ICM HH.

\begin{tabular}{lccccc}
\hline Classif./Refer. & Água & Várzea Baixa & Chavascal & $\begin{array}{c}\text { Várzea } \\
\text { Alta }\end{array}$ & $\begin{array}{c}\text { Soma/ } \\
\text { Linhas }\end{array}$ \\
\hline Água & 2111 & 0 & 0 & 0 & 2111 \\
Várzea Baixa & 0 & 1505 & 0 & 28 & 1533 \\
Chavascal & 0 & 83 & 1625 & 1318 & 3026 \\
Várzea Alta & 18 & 0 & 0 & 577 & 595 \\
\hline Soma/ Colunas & 2129 & 1588 & 1625 & 1923 & 7265 \\
Kappa $=0,7358$ & & & & & \\
Exatidão Global $(\%)=80,0826$ & & & & \\
Erro Global $(\%)=19,9174$ & & & & \\
\hline
\end{tabular}

Os resultados das classificaçóes MaxVer e ICM das componentes polarimétricas multivariadas em amplitude na banda L são apresentados na Figura 5. Na Tabela 5, são apresentados os valores estimados do coeficiente de concordância Kappa $(\hat{\mathbf{k}})$, as respectivas variâncias $\left(\hat{\boldsymbol{O}}_{\mathrm{k}}^{2}\right)$ e os conceitos obtidos segundo Landis e Koch (1977) para as classificaçóes MaxVer e ICM dos dados multivariados.

Considerando-se os dados bi e multivariados em amplitude das polarizaçóes na Banda L, e com base na análise das classificaçóes e respectivas matrizes de confusão, pode-se afirmar que:

Da mesma forma que para os dados univariados, quando comparadas às matrizes de confusão das classificações ICM, as matrizes de confusão das classificaçóes MaxVer apresentaram resultados inferiores para o índice de concordância Kappa, variância e Exatidão Global;

A combinação das três polarizaçóes e a combinação das três polarizaçóes adicionada à banda de textura receberam o conceito Excelente para a classificaçáo ICM, com índices Kappa de 0,8251 e 0,8963, respectivamente. A diferença entre as matrizes de confusão dessas duas combinaçóes (Tabela 6 e 7) diz respeito ao erro de inclusão para a combinação das três polarizações;

Tabela 5 - Exatidão das classificações dos dados bivariados e multivariados em amplitude na banda $L$ segundo 0 coeficiente de concordância Kappa $(\hat{\mathrm{k}})$ e variâncias $\left(\hat{\sigma}_{\mathrm{k}}^{2}\right)$.

\begin{tabular}{lccc}
\hline Classificação & $\hat{\mathbf{k}}$ & $\hat{\boldsymbol{O}}_{\mathrm{k}}^{\mathbf{2}}\left(\times 10^{-5}\right)$ & Conceito \\
\hline MaxVer HH-VV & 0,6515 & 4,5337 & Muito Boa \\
MaxVer HH-HV-VV & 0,6694 & 4,3978 & Muito Boa \\
MaxVer HH-HV-VV-TEX. & 0,7055 & 4,0785 & Muito Boa \\
ICM HH-VV & 0,7944 & 3,0197 & Muito Boa \\
ICM HH-HV-VV & 0,8251 & 2,6852 & Excelente \\
ICM HH-HV-VV-TEX. & 0,8963 & 1,7377 & Excelente \\
\hline
\end{tabular}

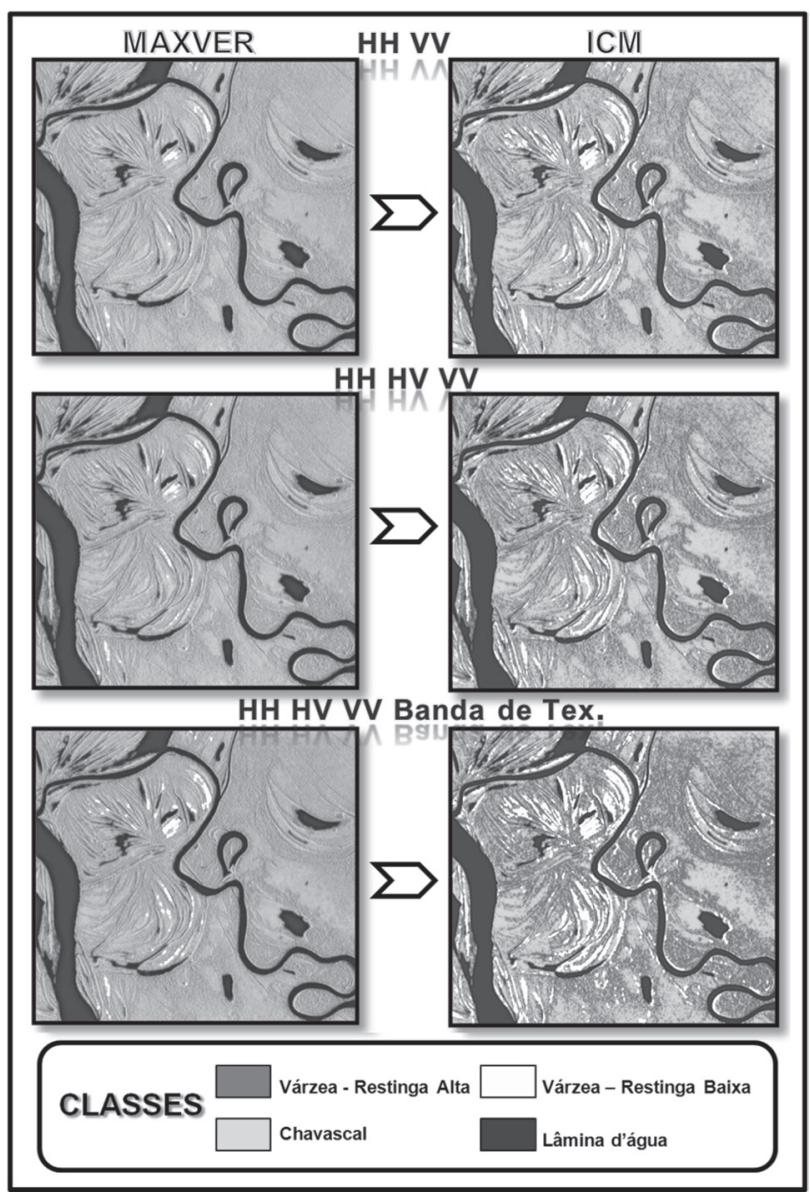

Figura 5 - Classificações geradas com os dados multivariados em amplitude.

A exatidão global da classificação ICM HH-HV-VVbanda de textura foi de 92,23\% (Tabela 7), demonstrando a efetiva melhora na classificação, em termos de exatidão do mapeamento, possibilitada pela adição da banda de textura que está associada a informaçôes relacionadas com a rugosidade da superfície dos alvos;

A utilização de dados bivariados e multivariados apresentou um ganho significativo em relação às classificaçôes com as polarizações individuais, confirmando os ganhos obtidos pelo sinergismo entre várias polarizaçôes e uso de dados derivados (ex. imagem textura).

$\mathrm{Na}$ área de estudo, nos locais onde a estrutura física do dossel florestal contém maior nível de biomassa vegetal (Floresta de Várzea - Restinga Alta), aparecem padrôes mais rugosos e bem definidos na superfície, possivelmente, devido à interaçấo de maior intensidade do dossel com as ondas de $23,5 \mathrm{~cm}$ da banda L. As áreas mapeadas com a fitofisionomia de Chavascal, predominantes em ecossistemas de Floresta de Várzea, caracterizam-se pela baixa biomassa e pelo contato 
Tabela 6 - Matriz de confusão resultante da classificação pelo algoritmo ICM, na imagem HH HV VV.

\begin{tabular}{lccccc}
\hline Classif./Refer. & Água & Várzea Baixa & Chavascal & Várzea Alta & $\begin{array}{c}\text { Soma/ } \\
\text { Linhas }\end{array}$ \\
\hline Água & 2116 & 0 & 0 & 0 & 2116 \\
Várzea Baixa & 2 & 1511 & 0 & 45 & 1558 \\
Chavascal & 0 & 71 & 1608 & 802 & 2481 \\
Várzea Alta & 11 & 6 & 17 & 1076 & 1110 \\
\hline Soma/Colunas & 2129 & 1588 & 1625 & 1923 & 7265 \\
Kappa $=0,8251$ & & & & \\
Exatidão Global $(\%)=86,8685$ & & & \\
Erro Global $(\%)=13,1315$ & & & & \\
\hline
\end{tabular}

direto entre solo e água, apresentando uma textura com pouca rugosidade, que é bem observada nos dados $\mathrm{SAR}$ em banda $\mathrm{L}$.

A variação dos parâmetros que envolvem a aquisição de uma imagem de radar pode atuar de maneiras diferentes em um mesmo alvo, provocando comportamentos diferenciados do retroespalhamento. Por outro lado, as variaçóes nas características morfológicas e estruturais do dossel também provocam comportamentos diferentes no retorno do sinal. Com os resultados obtidos, a partir dos processamentos envolvendo a combinação das três polarizaçôes adicionadas da banda de textura, principalmente para a Floresta de Várzea - Restinga Alta, gerando um realce da informação textural considerando-se a rugosidade e estrutura do dossel que contém a presença de espécies arbóreas de maior porte.

A reflexão especular da água evidenciou os cursos d'água e lagos na imagem investigada, justificando a interação das microondas que são refletidas especularmente na direção oposta da antena, e nenhum sinal é registrado, formando assim superfícies escuras. Por outro lado, devido ao efeito de reflexão de canto (double-bounce), as áreas com vegetação inundada foram realçadas, como por exemplo a fitofisionomia de Restinga Baixa que no momento de aquisição dos dados possuía um coluna d'água com cerca de 3,3 metros, ocorrendo assim um aumento na magnitude do sinal registrado pela

Tabela 7 - Matriz de confusão resultante da classificação pelo algoritmo ICM na imagem HH HV VV + banda de textura.

\begin{tabular}{lccccc}
\hline Classif./Refer. & Água & Várzea Baixa & Chavascal & Várzea Alta & $\begin{array}{c}\text { Soma/ } \\
\text { Linhas }\end{array}$ \\
\hline Água & 2113 & 0 & 0 & 0 & 2113 \\
\hline Várzea Baixa & 0 & 1553 & 12 & 61 & 1626 \\
\hline Chavascal & 0 & 23 & 1580 & 407 & 2010 \\
Várzea Alta & 16 & 12 & 33 & 1455 & 1516 \\
Soma/Colunas & 2129 & 1588 & 1625 & 1923 & 7265 \\
Kappa $=0,896314$ & & & & \\
Exatidão Global $(\%)=92,2367$ & & & \\
Erro Global $(\%)=7,7632$ & & & & \\
\hline
\end{tabular}

antena, caracterizado pela existência de níveis de cinza mais claros na imagem.

\section{CONCLUSÕES}

O sensor SAR R99 tem como principal vantagem a possibilidade de aquisiçáo dos dados em qualquer condiçáo atmosférica, considerando-se principalmente áreas da Amazônia. Entretanto, os algoritmos de processamento de dados SAR devem ser cada vez mais aprimorados, devido à exigência computacional atual.

A utilização de dados SAR em banda L do sensor aerotransportado R99 e algoritmos de classificação especificamente desenvolvidos para o processamento desse tipo de dados proporcionou uma classificação bastante precisa das fitofisionomias existentes na Floresta de Várzea da RDSA, da RDSM e áreas adjacentes. Estes resultados indicam o grande potencial dos dados SAR R99 em amplitude para distinção de classes de vegetação em ambientes alagados quando devidamente processados pelo algoritmo ICM.

A qualidade das classificaçóes obtidas com dados polarimétricos sob diferentes modelagens estatísticas foi avaliada quantitativamente através do coeficiente de concordância Kappa. Foram utilizados distintos tipos de dados SAR multi-look: univariados em amplitude (utilizando-se as distribuiçôes mais apropriadas), polarimétricos uni/bivariados, multivariados em amplitude (utilizando-se a distribuição normal mutivariada) e multivariados em amplitude. $\mathrm{O}$ uso das distribuiçôes multivariadas em amplitude, conjuntamente com uma banda de textura, produziu resultados de classificação de qualidade superior aos demais. O conceito Excelente foi obtido para esta classificaçáo segundo os intervalos de índice Kappa estabelecidos por Landis e Koch (1977). O resultado da classificação permitiu a discriminação correta de três classes vegetacionais de interesse, além da classe correspondente à lâmina d'água.

\section{AGRADECIMENTOS}

$\mathrm{O}$ primeiro autor agradece à Fundação de Amparo à Pesquisa do Estado de São Paulo (FAPESP) pela concessão da bolsa de doutorado, vinculada ao processo 04/06071-8. Os autores também prestam reconhecimento ao CENSIPAM pelo projeto aprovado e fornecimento dos dados SAR que possibilitou a realização do estudo. E também agradecem o apoio do Programa FEPIM 2004, financiado pelo Instituto de Desenvolvimento Sustentável Mamirauá - Projeto No 063-2004.

\section{BIBLIOGRAFIA CITADA}

Aboud Neta, S.R.; Freitas, C.C.; Dutra, L.V. 2010. Use of Multipolarized ALOS/PALSAR Image for Detection of 
Increment of Deforestation in the Amazon. Revista Brasileira de Cartografia, 62: 417-431. (in Portuguese, with abstract in English).

Ayres, J.M. 2006. Flooded forests in the Mamirauá: Middle Solimóes River. $3^{\mathrm{a}}$. ed. Sociedade Civil Mamirauá. 124 pp. (in Portuguese).

Correia, A.H. 1999. Project, development and evaluation of pointwise statistics and contextual classifiers for polarimetric SAR images. Dissertação de Mestrado, Instituto Nacional de Pesquisas Espaciais, São José dos Campos. 275 pp. (in Portuguese, with abstract in English).

Costa, M.; Niemann, O.; Novo, E.; Ahern, F.; Mantovani, J. 2002. Biophysical properties and mapping of aquatic vegetation during the hydrological cycle of the Amazon floodplain using JERS-1 and RADARSAT. International Journal of Remote Sensing, 23: 1401-1426.

de Jong, J.; Klaassen, W.; Ballast, A. 2000. Rain storage in forests detected with ERS tandem mission SAR. Remote Sensing of Environment, 72: 170-180.

Dutra, L.V.; Huber, R. 1999. Feature Extraction and Selection for ERS-1/2 InSAR Classification. International Journal of Remote Sensing, 20: 993- 1016.

Fernandez, G.A.V. 2007. Rate correlation between different polarizations of the L- and X-band SAR image acquired by the R99-B SIPAM seeking mapping logging, p. 7057-7062. In: Simpósio Brasileiro de Sensoriamento Remoto. Florianópolis, INPE. (in Portuguese, with abstract in English).

Freitas, C.C.; Soler, L.S; Sant'Anna, S.J.S.; Dutra, L.V.; Santos, J.R.; Mura, J.C.; Correia, A.H. 2008. Land-Use and Land-Cover Mapping in the Brazilian Amazon Using Polarimetric Airborne P-Band SAR Data. IEEE Transactions On Geoscience And Remote Sensing, 46: 2956-2970.

Gonçalves, F.G. 2007. Polarimetric SAR data analysis for tropical forest volume estimation. Dissertação de Mestrado, São José dos Campos, INPE. 109 pp. (in Portuguese, with abstract in English).

Gualberto, L.P.S.; Souza Filho, P.W.M. 2007. Recognition of coastal environments from textural analysis of R99B SAR images (L-band, VV polarization), p. 4889-4896. In: Simpósio Brasileiro de Sensoriamente Remoto, Florianópolis, INPE (in Portuguese, with abstract in English).

Guerra, J.B.; Mura, J.C.; Freitas, C.C. 2010. Discriminating deforestation increment areas in the Amazon rainforest with L band SAR R99B data. Acta Amazonica, 40: 557 - 566. (in Portuguese, with abstract in English).

Hess, L.L.; Melack, J.; Novo, E.M.L.M.; Barbosa, C.C.F.; Gastil, M. 2003. Dual-season mapping of wetland inundation and vegetation for the Central Amazon Basin. Remote Sensing of Environment, 87: 404-428.

Kasischke, E.S.; Melack, J.M.; Dobson, M.C. 1997. The use of imaging radars for ecological applications - a review. Remote Sensing of Environment, 59: 141-156.

Krohn, D.M.; Milton, N.M.; Segal, D.B. 1983. SEASAT synthetic aperture radar (SAR) response to lowland vegetation types in eastern Maryland and Virginia. Journal of Geophysical Research, 88: 1937-1952.

Landis, J.R.; Koch, G.G. 1977. The measurements of observer agreement for categorical data. Biometrics, 33: 159-174.

Le Toan, T.; Ribbes, F.; Floury, N.; Wang, L.F.; Ding, K.H.; Kong, J.A.; Fujita, M.; Kurosu, T. 1997. Rice crop mapping and monitoring using ERS-1 data based on experiment and modeling results. IEEE Transactions on Geoscience and Remote Sensing, 35: 41-56.

Lucas, R.M.; Moghaddam, M.; Cronin, N. 2004. Microwave scattering from mixed species woodlands, central Queensland, Australia. IEEE Transactions on Geoscience and Remote Sensing, 42: 2142-2159.

Miranda, F.P.; Beisl, C.H.; Camargo, E.C.G. 2007. Textural classification of R99 SAR data as an aid to flood mapping in Coari City, Western Amazon Region, Brazil, p. 4935-4942. In: Simpósio Brasileiro de Sensoriamento Remoto, Florianópolis, INPE. (in Portuguese, with abstract in English).

Narvaes, I.S.; Santos, J.R.; Silva, A.Q. 2010. Analysis of structural parameters of forest typologies using L-band SAR data. Boletim de Ciências Geodésicas, 16: 475 - 489.

Rosenqvist, A.; Shimada, M.; Chapman, B.; Freeman, A.; De Grandi, G.F.; Saatchi, S.; Rauste, Y. 2000. The global rain forest mapping project - a review. International Journal of Remote Sensing 21: 1375-1387.

Rosenqvist, A.; Milne, A.; Lucas, R.; Imhoff, M.; Dobson, C. 2003. A review of remote sensing technology in support of the Kyoto Protocol. Environment Science \& Policy, 6: 441-455.

Rosenqvist, A.; Shimada, M.; Chapman, B.; McDonald, K.; De Grandi, G.; Jonsson, H.; Williams, C.; Rauste, Y.; Nilsson, M.; Sango, D.; Matsumoto, M. 2004a. An overview of the JERS-1 SAR Global Boreal Forest Mapping (GBFM) Project. IGARSS'04, Anchorage, Alaska, USA, access September 20-24, 2004.

Rosenqvist, A.; Shimada, M.; Watanabe, M.; Tadono, T.; Yamauchi, K. 2004b. Implementation of systematic data observation strategies for ALOS PALSAR, PRISM and AVNIR-2. IGARSS'04, Anchorage, Alaska, USA, access September 20-24.

Rosenqvist, A.; Finlayson, C.; M., Lowry, J.; Taylor, D. 2007. The potential of long-wavelength satellite-borne radar to support implementation of the Ramsar wetlands convention Aquatic Conservation Maine Freshwater Ecosystem, 17: 229-244.

Saatchi, S; Agosti, D.; Alger, K.; Delabie, J.; Musinsky, Y.J. 2001. Examining fragmentation and loss of primary forest in the Southern Bahian Forest of Brazil with radar imagery. Conservation Biology, 15: 867-875.

Santos, J.R.; Narvaes, I.S.; Graça, P.M.L.A.; Gonçalves, F.G. 2009. Polarimetric responses and scattering mechanisms of tropical forests in the Brazilian Amazon, p. 183-206. In: G. Jedlovec (ed.). Advances in Geoscience and Remote Sensing. 1a. ed. Vukovar, Croatia.

SIPAM. 2007. ProAE-Monitoring Program Special Areas Identification of Deforestation on Indigenous Lands and Conservation Areas in the States of Acre, Mato Grosso and Rondonia - Technical Report. Centro Técnico Operacional de Porto Velho, Divisão de 
Monitoramento do Território, Porto Velho-RO, SIPAM/CTOPV - ProAE CD-ROM. (in Portuguese).

Ulaby, F.T.; Moore, R.K.; Fung, A.K. 1982. Microwave remote sensing: active e passive: Radar remote sensing and surface scattering and emission theory. $2^{\mathrm{a}}$ ed. Norwood, MA, Artech House. 1064 pp.

Vieira, P.R. 1996. Development of Maximum Likelihood and ICM classifiers for SAR images. Dissertação de Mestrado, Instituto de Nacional de Pesquisas Espaciais, São José dos Campos. (in Portuguese, with Abstract in English).
Wittmann, F.; Anhuf, D.; Junk, W.J. 2002. Tree species distribution and community structure of central Amazonian várzea forests by remote-sensing techniques. Journal of Tropical Ecology, 18: 805-820.

Recebido em 29/08/2010

Aceito em 11/01/2011 